\title{
Computer graphics in music performance research
}

\author{
CAROLINE PALMER \\ Ohio State University, Columbus, Ohio
}

\begin{abstract}
Theories of skilled music performance must account for variations on what is written in traditional musical notation. Some variations are intentional and reflect structural features of the music that are chosen for emphasis by the performer. Current music notations are inadequate to reflect these variations. Computer applications are described that allow graphical and statistical examination of performance variations on traditional musical notation. An integrated set of visual and sound tools is provided that allows music to be recorded, edited, analyzed, and played back on electronic and acoustic musical instruments. The graphical format allows flexibility through the use of windows, compression, expansion, and scrolling of multiple sources of information, mapping of acoustic to visual dimensions, and scaling of different performance parameters without normalization. Experimental evidence from piano performances is used to demonstrate how graphical formats can aid research on human performance.
\end{abstract}

A primary problem for theories of skilled music performance is to account for performed variations from what is written in music notation. Traditional music notation contains information on what note events should be played and when. However, human performance contains many variations in frequency, duration, timbre, and intensity. Some of these variations are intentional, and they are related to structural features of the music that are chosen for emphasis by the performer (Palmer, 1987; Rasch, 1979; Todd, 1985). For the purposes of research, current music notations do not adequately reflect these variations. Because a single musical performance contains much information (roughly $3 \mathrm{~K}$ bytes of information per minute of piano playing), researchers need better tools for extracting salient features from the complex relational information. In what follows, computer applications in music research are described that allow graphical and statistical examination of performance variations.

The graphical system described here combines familiar representations of musical knowledge with new but perceptually salient forms of representation. The goal is twofold: to make acoustic patterns perceptually salient in a visual domain, and to make available densely packed information at an appropriate level of resolution (MacKinlay, 1986). There are several reasons for this approach: first, whereas information in acoustic phenomena (such as music performance and speech) unwinds over time, up to a matter of minutes, information in visual

This research was supported by a predoctoral fellowship from the $\mathrm{Na}$ tional Science Foundation and by a research grant from the Psychology Department at Cornell University to the author. Acknowledgments are due Barry Vercoe, Michael Hawley, Robert Rowe, Gary Perlman, and the Music Cognition Group at the MIT Media Lab. Correspondence should be addressed to Caroline Palmer, Psychology Department, Ohio State University, Columbus, OH 43210. representations is available simultaneously. This decreases the selective auditory attention demands on the performer or listener. Second, visual representations allow the listener to directly contrast multiple excerpts or performances side by side, a comparison not possible acoustically. Finally, a visual representation allows examination of all details of the performance, both those easily heard and those not so easily heard.

A common approach to the study of variations in music performance is to characterize them in terms of structural aspects noted in the musical score, such as harmonic information, meter, and dynamics (intensity changes). Although this approach is helpful in modelling some variations in performance (Shaffer, Clarke, \& Todd, 1985; Sundberg, Askenfelt, \& Frydén, 1983), it does not address the variations between different performances of the same music. In other words, part of the variation is attributable to differences in individual performers' interpretations of structural content. Interpretations refer to musicians' individualistic modeling of a piece according to their own choices of appropriate musical structure for emphasis, such as melody, phrasing, and tempo changes (Apel, 1977). A successful graphical format must account for individual, as well as general, variations in musical expression.

The next section describes hardware and software for musical data collection, display, analysis, and performance. A subsequent section gives examples from a series of experiments on interpretation and expressiveness in piano performance (Palmer, 1987, 1988, in press). In these experiments, pianists were asked to verbalize and note on an unedited musical score their interpretation of the music-that is, any aspects of their performance not indicated in the notation that emphasize musical structure. Many performances of the same music are directly compared (in the graphical format) with each performer's 
structural interpretation; the goal is to find correspondences between the interpretations of structural content and the physical characteristics of performance. In the final section, graphical limitations and generalizations to other domains of human performance are discussed.

\section{Hardware and Software System}

The system is controlled by a Sun $3 / 260$ workstation, equipped with a greyscale monitor that can produce 256 levels of grey. The workstation is used as a host computer to control many musical instruments, including electronic synthesizers and an acoustic piano. The host computer controls the synthesizers through MIDI (Musical Instrument Digital Interface), an electronic communications standard representing a formal set of hardware and software rules for sending and receiving musical-event data between computers and musical instruments. Musicalevent data include pitch information, time of note onsets and offsets, and velocity information (correlated with loudness).

The host computer also controls a computer-monitored Bösendorfer Imperial Grand Piano. The piano contains optical sensors that monitor the time and velocity with which keys and footpedals are pressed. In addition, the piano contains solenoids that reproduce a performance on the piano with the same timing and velocity as during recording. The temporal resolution during recording and playback is $1 / 800$ th of a second $(1.25 \mathrm{msec})$. The hammer velocities constitute a range of 1,100 values, with an approximate sounded resolution of $0.1 \mathrm{~dB}$. The optical sensors and solenoids allow computer recording and playback without affecting the sound or touch of the piano.

The goal of the software environment is to provide an integrated set of graphical and sound tools that allow music to be recorded, edited, analyzed, and played back on the synthesizers and the grand piano. The graphical software includes a set of music sequencing operations with playback, record, cut, paste, and merge operations. ${ }^{1}$ Because traditional music notation cannot display the number of dimensions or the precision of each dimension adequately for research on musical performance, the software instead displays performances in the form of a piano roll shown on its side, with time increasing along the $x$-axis and pitch or frequency increasing along the $y$-axis. For keyboard instruments, foot-pedal information can be shown at the bottom of the display. An example of the graphical format is shown in Figure 1: a piano performance of a Chopin Prelude in D-flat Major (from Palmer, 1988).

Although the axes resemble traditional music notation, the rectangles and shading are new representations for displaying additional performance information. The rectangles represent precise note durations from onset to offset, and the shadings represent piano hammer velocities (correlated with loudness). The 1,100 possible velocity

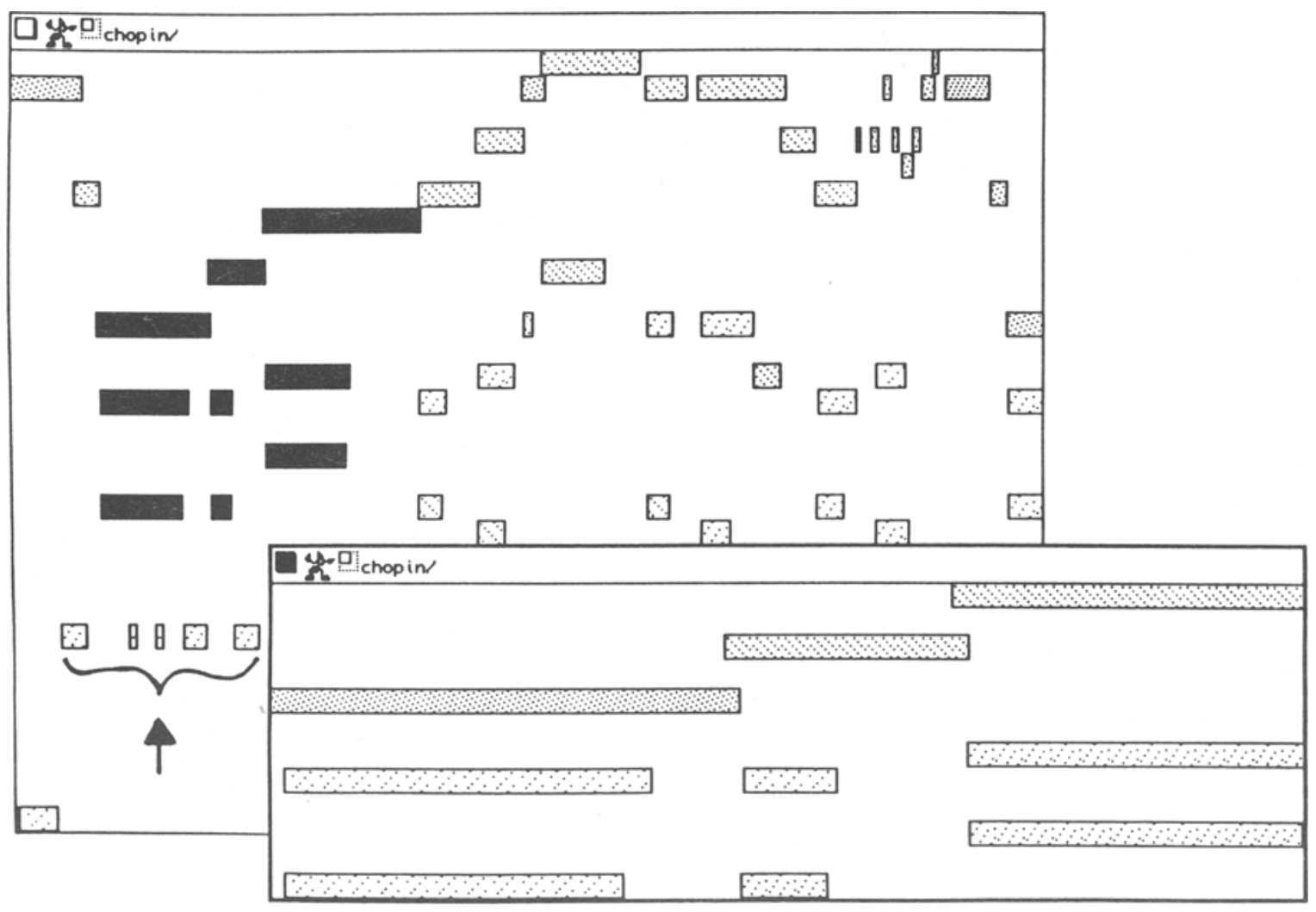

Figure 1. Graphical notation of piano performance of Chopin's Prelude in D-flat Major. Darkened notes are expanded in the inset box. 
levels are categorized into 16 greyscale levels to reflect the 45-50 dB range of loudness across performances (approximately $3 \mathrm{~dB}$ of increase per grey level). Thus, the graphic notation reflects perceptual constraints of sensitivity to changes in loudness on the order of $2 \mathrm{~dB}$ (Green, 1976). The performance in Figure 1 demonstrates some of the expressive variation from traditional notation; the arrow indicates a series of rectangles that, if played exactly according to the music notation, would all be of equal length (duration) and shade (loudness).

\section{Applications to Musical Performance}

One advantage of the graphical display is its flexibility, manipulated through the use of windows. Different performances or excerpts of performances can be assigned to different windows or portions of the computer screen, allowing separate manipulation of informational dimensions such as time or pitch. Depending on its configuration, the Sun workstation supports a large number of simultaneous window displays (at least 10 can be viewed at once). Traditionally, musical dimensions are compared across performances by normalization techniques (Bengtsson \& Gabrielsson, 1983). Window systems allow graphical comparisons without normalizing (the original values for each note event are retained); operations of compression and expansion are possible on any dimension (proportional alterations along a dimension in which the data are graphed). For example, performances of the same music can be aligned by pitch, and the original durations of each note are accessible by pointing the cursor at the note, causing the value to appear at the top of the window.

In addition, portions of a performance can be examined by sweeping a region of notes with the mouse, and selecting an option to create a new window containing just that region. Figure 1 demonstrates expansion of a region in a new window (the inset box). Scrolling, or shifting the data along an axis without compression or expansion, is available as well. Scrolling functions enable the relational information to be viewed at an appropriate level of detail without one's losing access to the whole. Windows, compression and expansion, and scrolling provide powerful tools for pattern recognition in complex time-series data such as music performances.

A second advantage of detailed graphical displays concerns the viewing resolution of densely packed information. This is especially important for music, because Western tonal music has been largely polyphonic over the last 600 years, consisting of two or more simultaneous parts or voices. The graphical software allows comparisons between densely packed musical parts, by displaying each note in a chord (events notated as simultaneous) in its temporal order. Small asynchronies are immediately apparent between notes that are not directly aligned vertically (in time). Although events are often notated by the composer as simultaneous, musicians rarely perform them simultaneously. As Rasch (1979) demonstrated with chamber ensemble players, the musician playing the melody or primary musical voice tends to precede the other players with a small lead. Asynchronies between events notated as simultaneous have also been measured in piano performance (Palmer, 1987, in press).

Figure 2 demonstrates asynchronies in two performances of the same musical excerpt from a Beethoven piano sonata, Opus 109 (from Palmer, 1988). The black notes indicate the voice intended by the performer as the melody. The inset boxes display the same musical location in each performance. In each of the inset boxes, the melodic notes tend to precede the other notes in each chord (vertically aligned events). Statistical procedures for analyzing the amount of asynchrony indicate that in most piano performances, the voice intended as melody by the performer precedes the remaining voices by $20-50 \mathrm{msec}$ (Palmer, in press), an amount large enough for correct perception of ordering (Hirsh, 1959). Only the voice intended as melody by the performer displays this asynchrony. Furthermore, when pianists were asked to play an unmusical or flat, mechanical performance of the same excerpt, the melody lead decreased, and in an exaggerated performance the melody lead increased (Palmer, in press). These findings suggest that the asynchronies may be a performance cue for perceptually separating the melody from remaining events notated as simultaneous.

A third advantage of this system concerns event-oriented changes such as musical dynamics or loudness patterns. Traditional music notation displays only occasional changes in loudness across large structures such as phrases, but performers apply changes in dynamics to each note event. Graphical systems can display that information. Because of the greyscale monitor capacities of the Sun workstation, differences in how hard each note is hit on a piano keyboard can be displayed graphically by means of shading each note on a white-to-black (quiet-to-loud) dimension. Thus, the visual intensity contours represent acoustic intensity contours. Although this system employs greyscale levels to display event-oriented informational changes, other monitor capabilities such as color or black and white can be implemented in a similar manner.

Event-oriented changes in loudness are shown in Figure 3 in two performances by one pianist of the same musical excerpt from a Brahms Piano Intermezzo, Opus 118 No. 2 (from Palmer, 1988). The inset boxes display the same musical location in each performance. The lines indicate the pianist's intended phrasing. The graphics system demonstrates a correspondence between pianists' intended phrase structure and loudness contours. Note events at the endings and beginnings of adjacent phrases (as notated by the pianist) coincided with large changes in loudness, indicating that pianists use changes in loudness to signal phrase structure by marking the boundaries of event groups (Palmer, 1988).

Other signals of intended phrasing evident in this graphical notation are the changes in rubato and large temporal gaps at phrase boundaries. By rubato I mean note-to-note changes in tempo or rate, which are typically largest at 

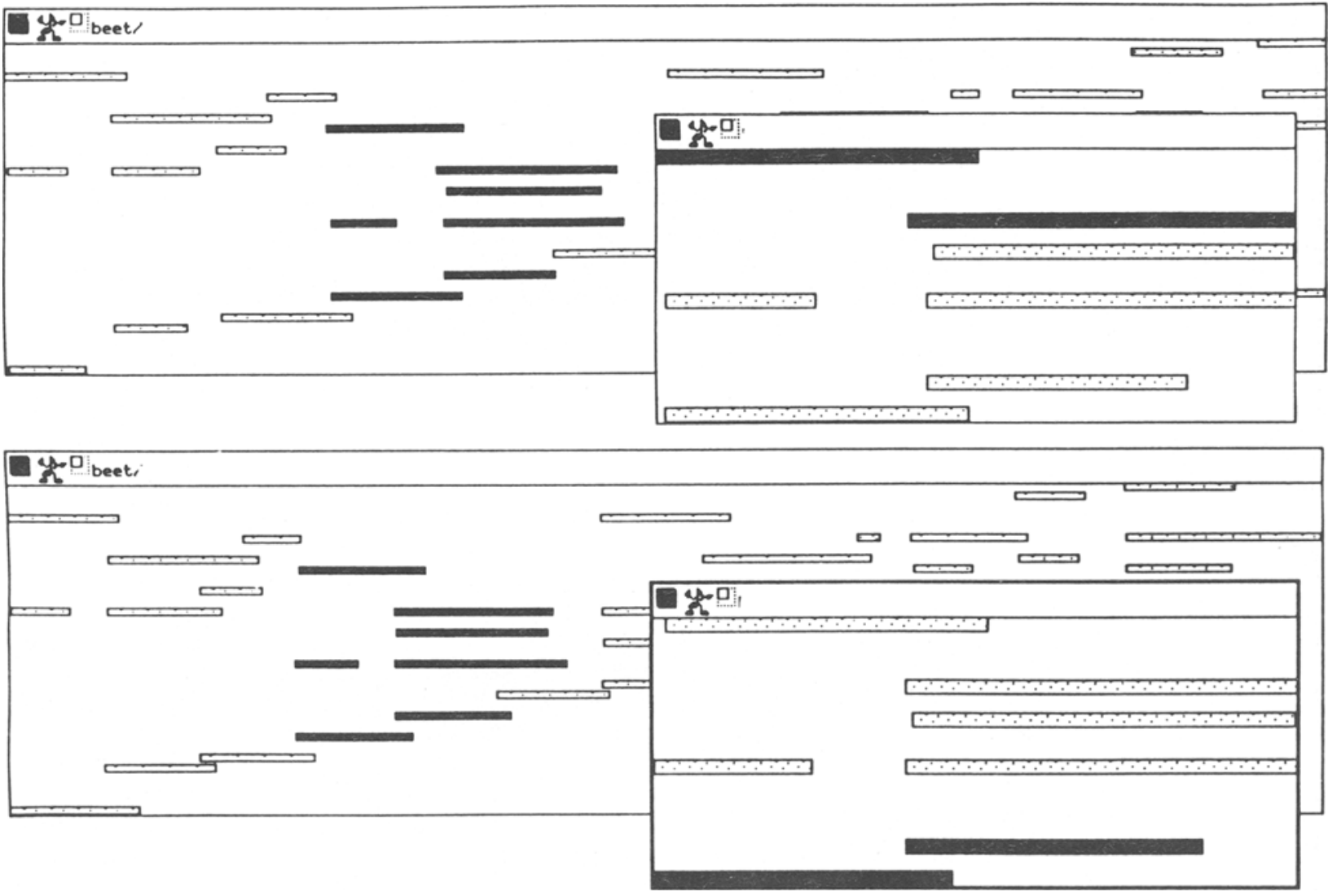

Figure 2. Two performances by same pianist of theme from Beethoven's Piano Sonata in E Major, Opus 109. Inset boxes display chord asynchronies between intended melody (in black) and remaining voices.

phrase boundaries. In speech and music, pausing and increasing the durations of events at phrase boundaries are assumed to be accounted for by syntactic and prosodic structure (Liberman \& Prince, 1977; Todd, 1985). Algorithms have been developed that predict pausing by an amount proportional to the hierarchical level or depth of phrase embedding (Cooper \& Paccia-Cooper, 1980; Grosjean, Grosjean, \& Lane, 1979; Todd, 1985). These algorithms predict tempo changes only from information in the notation, not from the individual performer's intentions regarding structural content. Visual analysis allows examination of individualistic phrasing effects in music, through the comparison of each performer's intentions with the durations of note events. As demonstrated in Figure 3, the location and amount of phrase marking are directly related to the pianist's phrasing interpretation. The relative durations of the note events indicate tempo changes greater at phrase boundaries than within phrases, but only when performances are viewed in terms of the intended interpretation (Palmer, 1988, in press). This suggests that some of the variation in performance can be accounted for by each performer's intended syntactic structure.

Another benefit of graphical systems concerns relations among temporally adjacent events. Examples include legato and staccato, techniques common to the performance of music. They are not usually depicted in traditional music notation, but rather added by the performer. Legato and staccato refer to the amount of temporal overlap between adjacent note events. The arrows in the top portion of Figure 4 show examples of legato (L) and staccato (S). Legato is a smooth, continuous flow of notes, caused by the offset of one note following the onset of the next note. Staccato is an abrupt, discrete patterning of notes, caused by gaps or nonoverlapping offsets and onsets. Legato and staccato patterns, or overlaps, are thought to create perceptual continuity or discontinuity in a melodic line or voice.

In a learning study, pianists were asked to sight-read or perform an unfamiliar musical excerpt, and successive performances by each pianist were recorded and compared (Palmer, 1988). Shown in Figure 4 are the 1st (top) and 10th (bottom) performances by one pianist of the opening theme from a Beethoven Bagatelle, Opus 126 No. 1 . As can be seen, the 10th performance shows more legato (positive overlap) than the $1 \mathrm{st}$ performance. As the pianist became more familiar with the work, the amount of legato increased. Statistical analyses indicated that the amount of legato or staccato was proportional to the durations of the surrounding note events (Palmer, 1988, in 


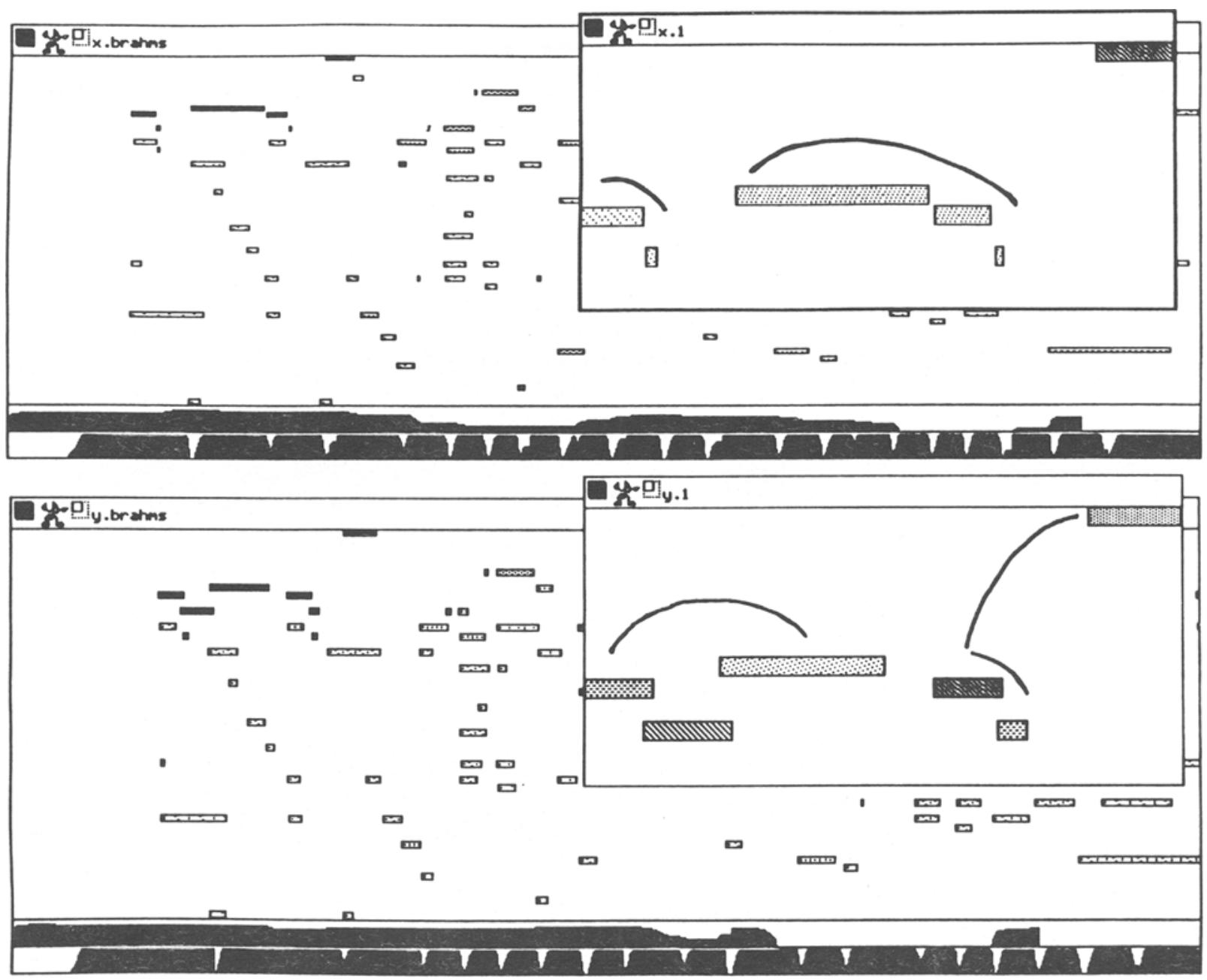

Figure 3. Two performances of the same opening section of Brahms' Piano Intermezzo, Opus 118 No. 2. Inset boxes display tempo changes coincident with pianist's intended phrasing (indicated by lines). Bottom areas in each window display soft pedal (top line) and sustain pedal (bottom line); gaps indicate pedal release.

press). Only when the pianist's intended melody and phrasing were considered were the overlaps proportional to the note durations. This supports the conclusion that overlaps reflect an interpretive aspect, determined by intentions of musical expression.

Graphical performance systems offer other advantages as well. They aid in music synthesis with the cut, paste, and merge options. These syntheses can be played for listeners, to evaluate the validity of the different performance cues extracted from the graphical and statistical analyses. The distribution of expressive parameters can differentially affect the perceptual salience of musical events and the successful communication of the performer's interpretation to a listener (Palmer, 1988). The graphical and statistical analyses described here can aid in modeling the assignment of musical meaning to skilled performance.

\section{Conclusions}

Traditional musical notation has remained relatively constant over the last 300 years. However, new compositional and sound-synthesis techniques, as well as research in music performance, require more detailed graphical notations. The system described here does not address many elements of musical performance, such as changes in timbre and frequency, and differences between particular musical instruments. However, it is not difficult to envision a similar graphical system that would incorporate these dimensions. For instance, timbre might be encoded with color or by means of stacking symbols along a third dimension (similar to graphical displays of Fourier analyses). Frequency changes could be encoded along the vertical dimension through alteration of the symbol shape (curved lines could be used instead of rectangles). It is likely that the expression of musical meaning in perfor- 

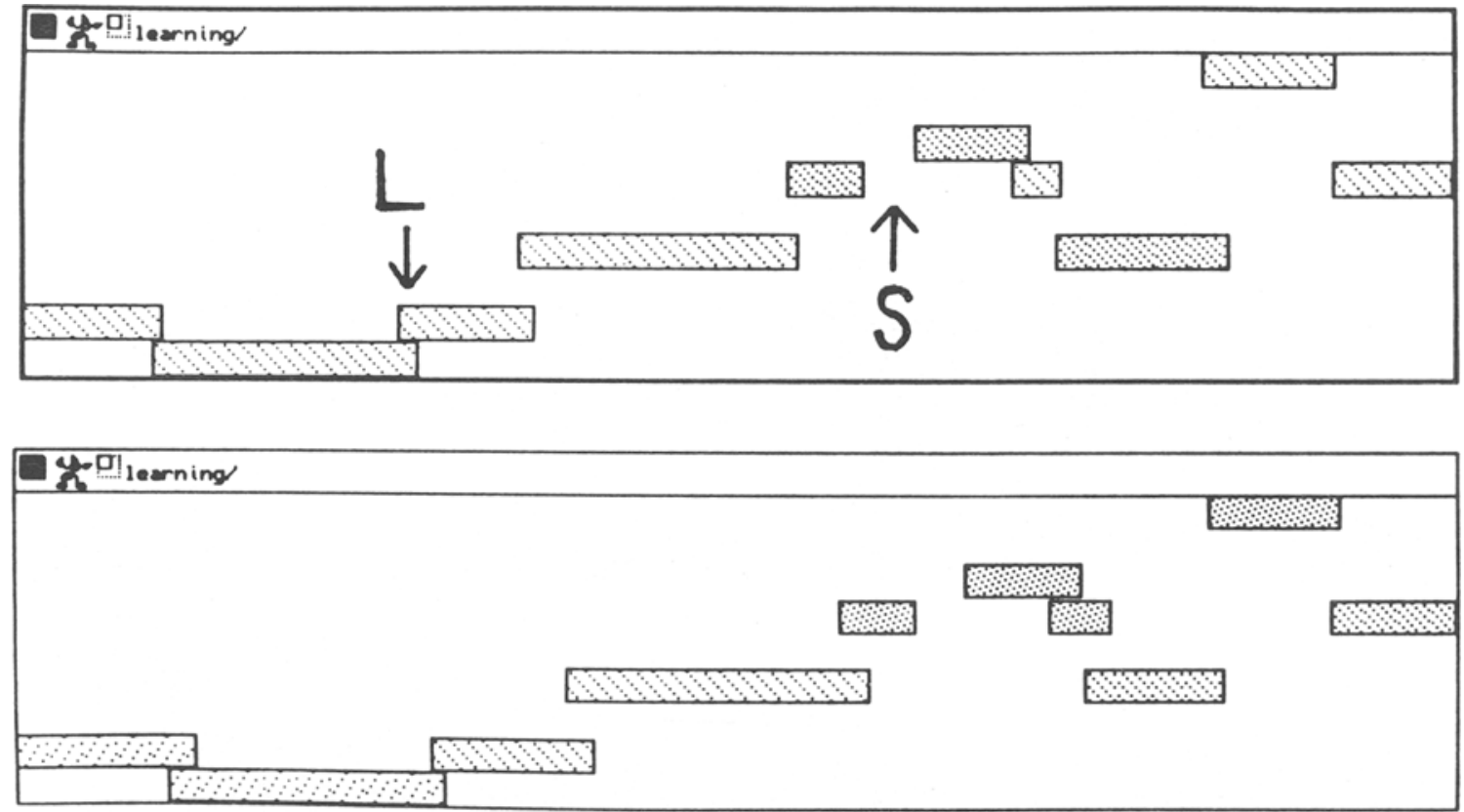

Figure 4. Melody from two performances by same pianist of an unfamiliar excerpt (opening theme from Beethoven's Bagatelle, Opus 126 No. 1). Top: Ist performance. Bottom: 10th performance. Arrows indicate examples of legato (L) and staccato (S).

mance shows some generalities across musical dimensions and instruments, based on human perceptual and representational constraints. An efficient graphical design should mirror those generalities.

The primary goal of new graphical notations is to make available large amounts of information and allow manipulation of that information. This goal is best approached, I believe, through the creation of representational formats that allow an appropriate level of detailed analysis and that are designed in accordance with the limits of human perceptual capacities. Similar graphical systems might apply to other types of time-based human performance such as dance; once the dimensions of interest have been determined (such as limb or body movements), the graphical parameters can be assigned (such as color and shape), and the same visual tools of windows, compression and expansion, and scrolling can aid in modeling human performance.

\section{REFERENCES}

APEL, W. (1977). Harvard dictionary of music. Cambridge, MA: Harvard University Press.

Bengtsson, I., \& Gabrielsson, A. (1983). Analysis and synthesis of musical thythm. In J. Sundberg (Ed.), Studies of music performance (pp. 27-60). Stockholm: Royal Swedish Academy of Music.

Cooper, W. E., PaCcia-CoOper, J. (1980). Syntax and speech. Cambridge, MA: Harvard University Press.

GreEN, D. M. (1976). An introduction to hearing. Hillsdale, NJ: Erlbaum.
Grosjean, F., Grosjean, L., \& Lane, H. (1979). The patterns of silence: Performance structures in sentence production. Cognitive Psychology, 11, 58-81.

HiRSH, I. J. (1959). Auditory perception of temporal order. Journal of the Acoustical Society of America, 31, 759-767.

Liberman, A. M., \& Prince, A. (1977). On stress and linguistic rhythm. Linguistic Inquiry, 8, 249-336.

MaCKINLAY, J. (1986). Automating the design of graphical presentations of relational information. ACM Transaction of Graphics, 5, 110-141.

Palmer, C. (1987). Effects of interpretation on timing in piano performance. Journal of the Acoustical Society of America, 81, S92.

Palmer, C. (1988). Timing in skilled music performance. Unpublished doctoral dissertation, Cornell University, Ithaca, New York.

PALMER, C. (in press). Mapping musical thought to musical performance. Journal of Experimental Psychology: Human Perception \& Performance.

RASCH, R. A. (1979). Synchronization in performed ensemble music. Acustica, 43, 121-131.

Shaffer, L. H., Clarke, E. F., \& Todd, N. P. (1985). Metre and rhythm in piano playing. Cognition, 20, 61-77.

SundBerg, J., Askenfelt, A., \& Frydén, L. (1983). Musical performance: A synthesis-by-rule approach. Computer Music Journal, 7, $37-43$.

ToDd, N. P. (1985). A model of expressive timing in tonal music. Music Perception, 3, 33-59.

\section{NOTE}

1. The graphical software was first developed by Michael Hawley and Robert Rowe of the Music Cognition Group, MIT Media Lab, Cambridge, MA 02139 . 